\title{
Design of Radar Countermeasure and Anti-Countermeasure Simulation Training System
}

\author{
Ming LI \\ Ordnance Engineering College, Shijiazhuang 050003, P. R. China \\ liming_fly@126.com
}

\begin{abstract}
Keywords: radar reconnaissance reconnaissance and interference, radar anti-jamming, simulation training system, effect evaluation

Abstract. Based on the analysis of the functional requirements of radar confrontation and anti-anti-simulation training system, a simulation training system based on distributed local area network is designed. Practice has proved that the design is complete, mature technology, stable operation, can independently form the training required battlefield environment and electromagnetic situation, similar simulation training equipment design and development of reference.
\end{abstract}

\section{Introduction}

Radar confrontation and anti-anti-simulation training system's main functions include: First, simulate the radar against the equipment work process, radar reconnaissance and radar interference related operations, simulation related processes and effects; the second is to simulate the radar equipment work process, simulated radar interference The basic operation and radar anti-jamming operation, through the use of a variety of anti-jamming measures to reflect the effect of different anti-jamming measures; Third, the radar interference and anti-jamming effects to assess.

\section{Simulation training system composition and working principle}

Radar confrontation and anti-simulation training system for pure software simulation training system, the main functional modules include: guidance control subsystem, radar operation training simulator, radar combat training simulator and run support environment, radar and anti-anti-simulation The structure of the training system is shown in Fig.1.

System operation flow: The guidance control subsystem according to the training needs, set the simulation parameters, start the simulation and simulation control; guidance control subsystem in the simulation process to simulate the flight targets and publish; radar operation training simulator for radar switch and Radar attack operational training simulator to detect the radar signal, according to the reconnaissance results of the radar interference, announced interference parameters; radar operation training simulator according to the interference parameters in the terminal display interference phenomenon, the operator to carry out the radar Anti-interference operation; during the confrontation process, the guidance control subsystem can also provide the background of the electromagnetic environment signal and confront the trend display; confrontation after the guidance of the control system can be based on training process data to assess the effectiveness of training.

The simulation training system adopts red, blue and white countermeasure mode, which can reflect the confrontation in the simulation system and solve the problem of the simulation system management function. White is the steering control subsystem, responsible for the simulation system management and control. In the simulation process, it is responsible for process control, clock synchronization, information exchange, target control, situation display, and collecting recorded simulation information. After the simulation, according to the simulation, the simulation is carried out. The information collected is evaluated for training effectiveness. Radar / radar counterfeit parties are responsible for simulating their own equipment confrontation: in the simulation preparation phase, according to the simulation parameters received from the white side; in the simulation process, 
according to the status of both sides of the operation of the enemy, to develop offensive and defensive strategy, control their own equipment to fight.

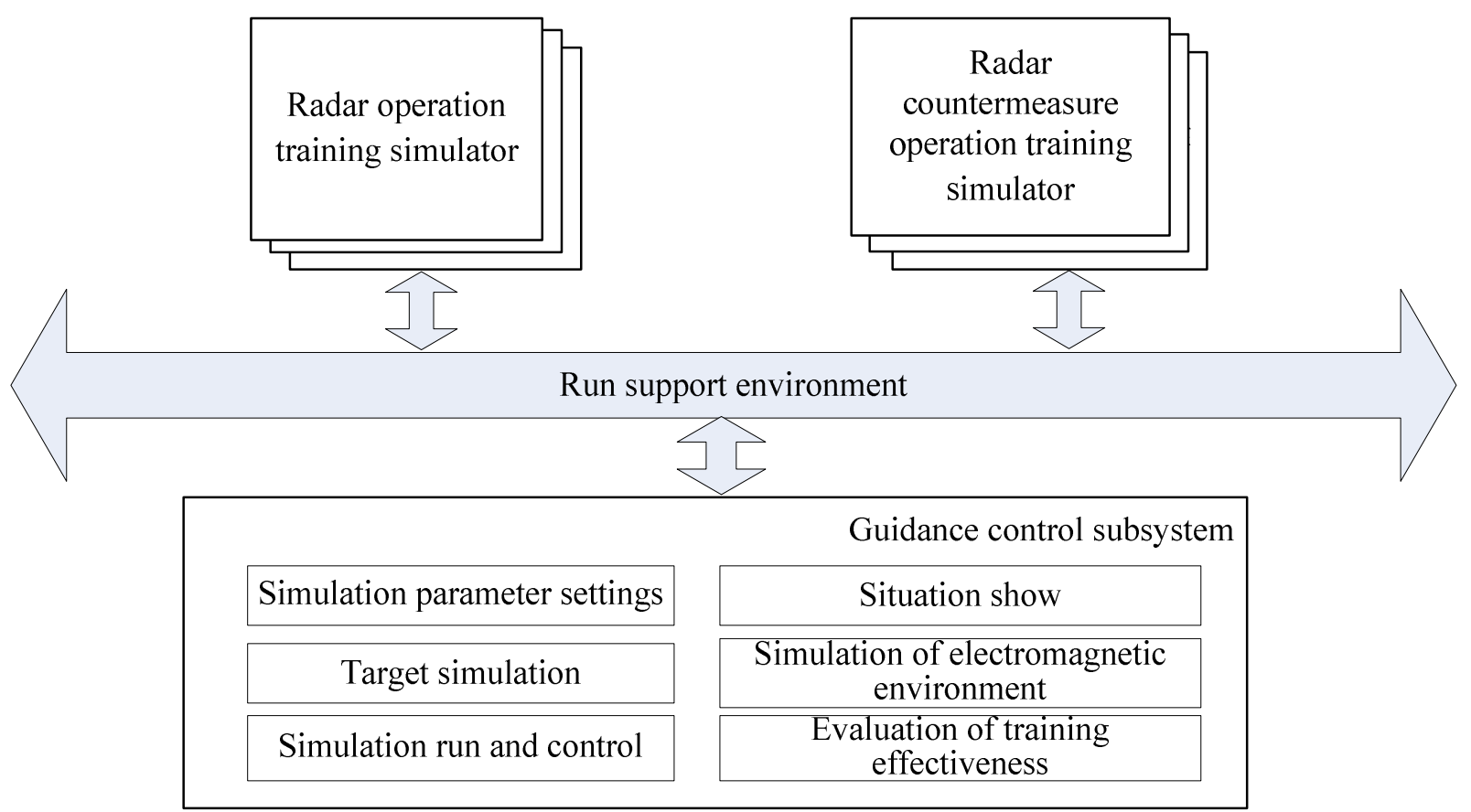

Fig.1 Radar confrontation and anti-anti-simulation training system structure

\section{Simulation training system implementation}

(1) Guidance control subsystem

The guidance control subsystem includes functions such as simulation parameter setting, target simulation, simulation operation control, situation display, electromagnetic environment simulation and training effect evaluation.

Simulation parameters set the main completion of the system radar equipment, radar equipment, target-related parameters set. Among them, the radar-related parameters include: equipment type, location and other parameters of the deployment. Radar countermeasure equipment related parameters include: the initial location, flight routes, motor speed and so on. Target-related parameters include: target batch, target radar cross-sectional area, maneuvering speed, maneuvering route and so on. The target simulation mainly completes the control of the flight target, and provides the target condition for the radar search and tracking operation. The simulation run control mainly realizes the control and monitoring of the simulation process. Can provide appropriate control management components, the simulation process management, control and monitoring. The situation shows the main completion of the confrontation of the show, to achieve the visualization of the simulation process. Using switching, stratification, window, zoom, roaming, etc., both a comprehensive demonstration of the battlefield electromagnetic situation, but also a site or a battlefield on a local scene. The purpose of the electromagnetic environment simulation function is to simulate the background electromagnetic signal environment. The training effect evaluation is mainly to evaluate the effect of radar interference and anti-jamming. During the simulation operation, the original data and simulation operation data are collected automatically, and the radar effect is evaluated by mathematical model. The evaluation function can record each operation of the operator, and use the reasoning mechanism and the deductive mechanism to compare with the best method and procedure. The combination of the length of time, the choice of the interference means, the operation 
state judgment, the logical relationship of the operation information, The weight of the factors, give a reasonable assessment of the results.

(2) Radar operation training simulator

Radar operation training simulator mainly in the form of software to achieve virtual radar equipment. Radar operation training simulator and radar in the layout of the layout, the color is consistent, the working principle of the simulator, working process, working conditions, timing and other consistent with the real, simulator external communication interface and radar confrontation and anti-anti-simulation training The rest of the system is consistent. Virtual radar equipment can be used to carry out basic radar training and anti-jamming training.

Radar operation training simulator features include: First, the basic operation of the simulation radar training process, including radar start training, radar shutdown training, radar search target training, radar tracking target training; the second is simulated radar interference phenomenon, in the radar interface On the display; Third, the simulation radar to adjust the operating parameters, reduce the probability of being intercepted to eliminate the process of electronic interference. In the form of functional simulation, the radar control and tracking process simulation are carried out for the typical fire control radar and target indicator radar. The signal recovery and local signal simulation are used to simulate the radar interference.

First, the radar boot operation; and then order the guidance control system to provide the target information, radar search tracking training, published radar work parameter information; training process, the order of radar operation training simulator information to determine whether the radar is disturbed, if Interference is shown to interfere with the phenomenon, as a basis for taking anti-jamming measures. Whether the radar is disturbed can be judged from four aspects: time condition, frequency condition, space condition and energy condition.

Time delay: the instantaneous bandwidth of the radar in the instantaneous bandwidth of the interference signal transmission (interference frequency coverage radar frequency), the frequency of the radar, ; Space conditions to determine: radar jammer main lobe to the radar; energy conditions to determine: the main lobe interference, into the radar receiver interference power and target echo power is greater than the radar suppression factor.

(3) Radar Countermeasure System Operation Training Simulator

Radar countermeasure system operation training simulator is mainly used in the form of software to simulate the typical radar confrontation system display and operation interface, and has the following functions: First, simulated radar against the radar signal reconnaissance process, get reconnaissance results, display Reconnaissance parameters, including the number of signs, azimuth, carrier frequency, carrier frequency type, frequency, frequency type, pulse width, pulse width type, pulse amplitude, threat level, etc .; Second, the radar interference control, according to reconnaissance As a result, the interference parameters are set to interfere with the radar. During the interference process, the radar batch number which can be disturbed can be displayed, and the working state of the jammer is displayed, including interference pattern selection, interference parameter setting and interference parameter.

Reconciliation conditions are considered taking into account the following factors: airspace conditions, frequency domain conditions and energy domain conditions. Airfield conditions refer to radar reconciliation reconnaissance systems that should be consistent with radar direct and beam alignment; frequency domain conditions refer to radar signal frequencies within the operating frequency range of radar reconciliation reconnaissance systems; energy domain conditions refer to radar confrontation The radar signal received by the reconnaissance system should be greater than the minimum detectable signal power of the system. When the three factors all meet the conditions, you can determine the radar reconnaissance reconnaissance system can detect the radar signal. 


\section{Conclusions}

Practice has proved that the simulation training system is reasonable in design, realistic and cost-effective. Due to the modular design, it has good scalability and reorganization ability, which satisfies the corresponding demand for dressuping. At the same time, the mature technology in this design is of great significance to the development of other similar analog training systems.

\section{References}

[1] Tang see soldiers, Zha Ya soldiers. Combat simulation system verification, verification and confirmation and credibility assessment [M]. Beijing: National Defense Industry Press, 2013: 133-183.

[2] Chai Yuanbo, Wang Yueqing. Introduction to battlefield information confrontation [M]. Beijing: National Defense Industry Press, 2009: 109-176.

[3] (US) Stephen J Andriole. Advanced system of command system engineering [M]. Beijing: National Defense Industry Press, 2005: 83-164.

[4] Luyue Guang, Fang Shengliang. Combat Experiment [M]. Beijing: National Defense Industry Press, 2007: 186-318.

[5] Dong Zhiming, Guo Qisheng, Huang Xiying, and so on. Battlefield environment modeling and simulation [M]. Beijing: National Defense Industry Press, 2013: 163-204.

[6] Wang Yang. Simulation of anti - noise interference of main passive composite radar seeker [D]. Changsha: National University of Defense Technology, 2011. 